\title{
Irradiation Tests of Photodiodes for the ATLAS SCT Readout
}

\author{
J.D.Dowell, R.J.Homer, I.R.Kenyon, G.Mahout, S.J.Oglesby, H.R.Shaylor, \\ J.A.Wilson. \\ School of Physics and Astronomy, \\ University of Birmingham, \\ Birmingham. B15 2TT. \\ R.Nickerson, R.Wastie, A.Weidberg. \\ Nuclear and Astrophysics Laboratory, \\ University of Oxford, \\ Keble Road, Oxford. OX1 3RH.
}

\begin{abstract}
Bulk and epitaxial Si PIN diodes were exposed to a fluence of around $2.5 \times 10^{14}$ $\mathrm{cm}^{-2} 1 \mathrm{MeV}$ equivalent neutrons followed by a fluence of approximately $2.5 \times 10^{14}$ $\mathrm{cm}^{-2} 24 \mathrm{GeV}$ protons.

The DC response of the devices showed a degradation after the first $\sim 0.25 \times$ $10^{14} \mathrm{~cm}^{-2}$ of neutron fluence, but then no further degradation. The response of the bulk devices degraded markedly to only $10 \%$ of their initial response, whereas the epitaxial devices response degraded to $\sim 70 \%$ of their initial value, a level still acceptable for their use in binary links.

Both types of diode exhibited a change in dark current related to the incident $1 \mathrm{MeV}$ equivalent neutron fluence, $\phi$, that can be described by the equation $\Delta I_{d c}=$ $K \phi$. The characteristic damage constants are $K=3.3 \times 10^{-12} \mathrm{nA} \mathrm{cm}^{2}$ and $K=$ $0.13 \times 10^{-12} \mathrm{nA} \mathrm{cm}^{2}$ for the bulk and epitaxial diodes respectively.

Finally, the AC response of an epitaxial Si PIN diode was measured after irradiation. Both the rise and fall times were measured to be less than 1 ns for a bias voltage greater than $5 \mathrm{~V}$.
\end{abstract}




\section{Introduction}

Crucial to the use of any electronic components in the inner tracker regions of Large Hadron Collider (LHC) experiments is the understanding of their performance after irradiation with neutral and charged hadrons and gammas. In the ATLAS Semiconductor Central Tracker (SCT) the predicted fluences over a ten year operational life are $\sim 10^{14}$ neutrons $\mathrm{cm}^{-2}$ and $\sim 10^{14}$ charged-hadrons $\mathrm{cm}^{-2}$. The predicted ionizing dose is $\sim 10 \mathrm{MRad}$.

The readout architecture for the baseline ATLAS SCT link [1,2] is shown in figure 1. The front-end hybrid board connects to the detector and holds the frontend binary readout chips. A separate opto-hybrid holds the optical components.

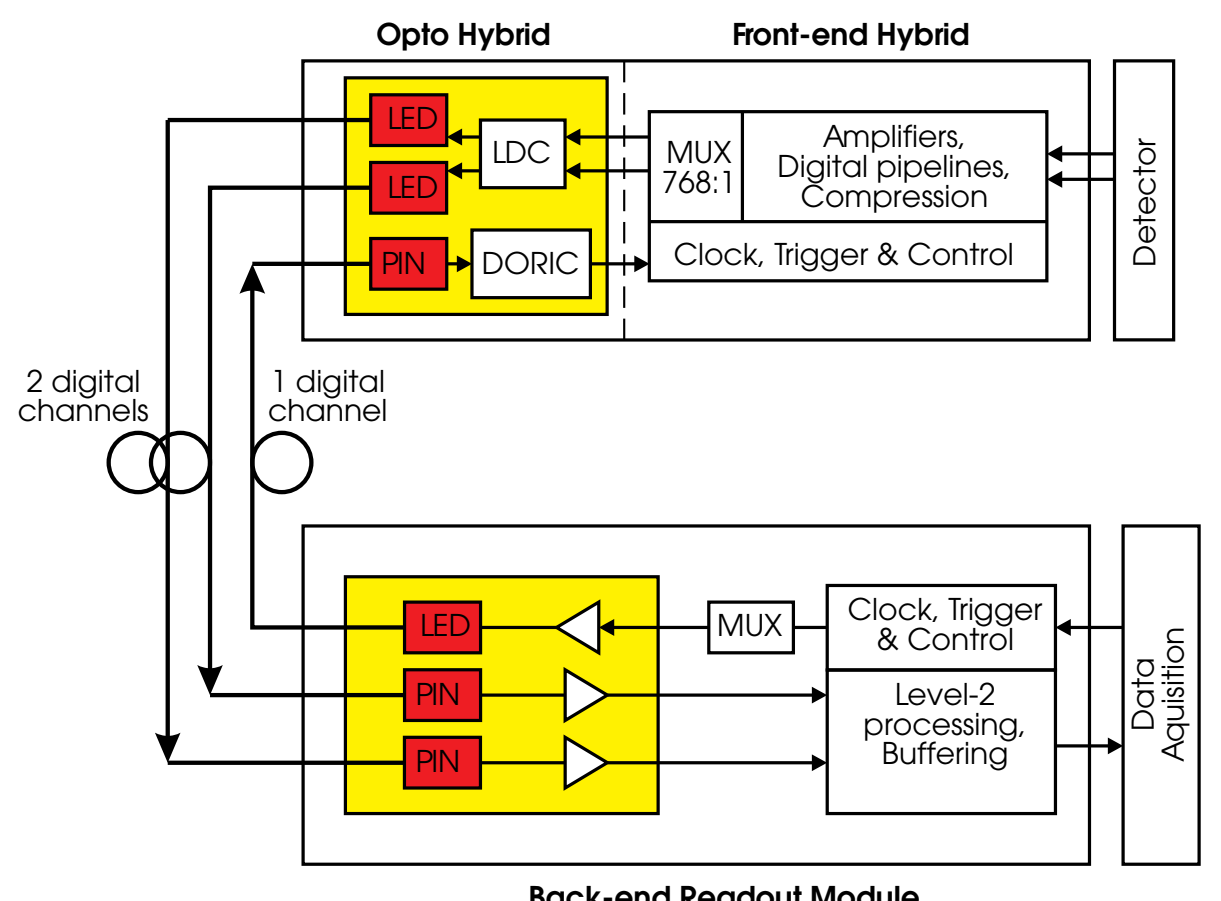

Figure 1: Schematic of the proposed binary optical readout system for the ATLAS SCT.

The opto-electronic package used in the binary readout data link is shown in figure 2. The same package is also under consideration for use in the readout of data from the pixel detector. Locating the optical components at the outer barrel pixel layer (radius $11 \mathrm{~cm}$ ) would require tolerance to fluences approximately five times those in the SCT.

The opto-electronic package is custom-designed and manufactured by GECMarconi, UK(a). It contains the two LEDs, used for the readout of the binary front-end data, and the PIN photodiode that receives the bi-phase mark encoded timing, trigger, and control (TTC) data required by the module. It also provides ground planes for the mounting of the LED driver chip (LDC) and TTC decoder

(a) GEC Part no. 520/1/02113/000. 
chip (DORIC). The packaging is specifically designed to be low-mass, non-magnetic and radiation hard to match the $\mathrm{SCT}$ requirements.

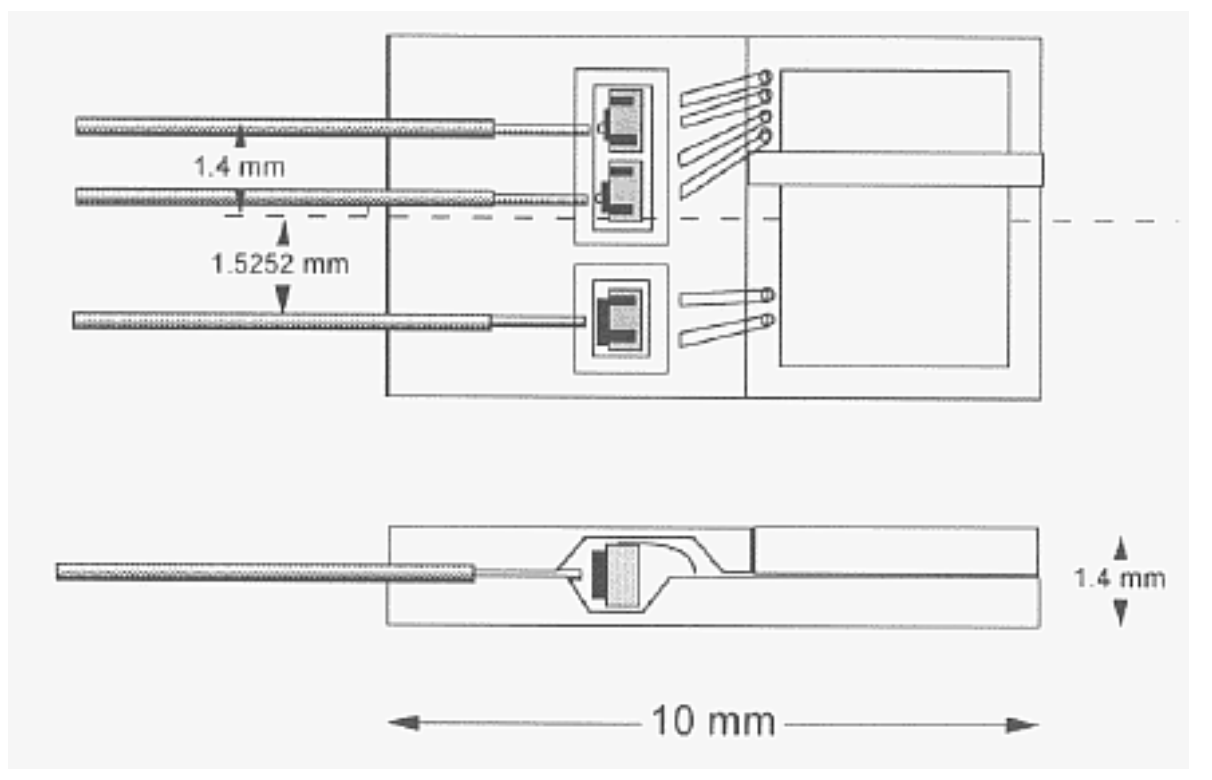

Figure 2: Diagram of the custom GEC LED/PIN package.

The performance of the LEDs and also VCSELs after irradiation has been studied $[3,4]$ and the LEDs have been shown to be sufficiently radiation hard for use in the SCT, whereas VCSELs are extremely radiation hard and would be suitable for use in the pixel detector. Presented in this report are measurements of the performance of photodiodes after irradiation with neutrons and protons.

Two types of photodiode have been investigated for use in the package:

Bulk silicon photodiodes ${ }^{(b)}$ were used in the first prototype packages but are not adequate for the final system. Their unirradiated response, $\sim 0.5 \mathrm{~A} / \mathrm{W}$, is sufficient; however the $10 \%-90 \%$ rise and fall times of $15 \mathrm{~ns}$ at low bias voltage $(-5 \mathrm{~V})$ are inadequate for $40 \mathrm{Mbit} / \mathrm{s}$ operation. They are made from a low-doped background n-type Si substrate. Dopants are then diffused into this substrate to form a thin p-type layer at the top of the device and a thin n-type layer at the bottom. The light is incident upon the device from the top. The chip thickness, and hence the depletion region thickness is $\sim 100-150 \mu \mathrm{m}$;

Epitaxial silicon photodiodes ${ }^{(c)}$ exhibit a lower, but still acceptable, response $(\gtrsim 0.35 \mathrm{~A} / \mathrm{W})$ and the faster rise and fall times $(\lesssim 1 \mathrm{~ns}$ at $-5 \mathrm{~V}$ bias) required for operation at $40 \mathrm{Mbit} / \mathrm{s}$. They are made from an n-type substrate which then has a low-doped background (intrinsic) n-type layer followed by a ptype layer grown epitaxially on top. The low-doped region which forms the depletion layer is only $\sim 15 \mu \mathrm{m}$ thick. Therefore the epitaxial devices can

(b) GEC Part no. 520/1/02603/000.

${ }^{(c)}$ GEC Part no. 520/1/02603/001. 
be fully depleted at very low bias voltages even after radiation damage has increased the carrier concentrations.

\section{Radiation Damage Theory}

The performance of a photodiode as a detector can be characterised by three parameters:

- The dark current is the leakage current when there is no light incident on the photodiode;

- The responsivity of the photodiode describes its efficiency in converting light into an electrical signal, measured in $A / W$;

- The rise and fall time of the photodiode limits its speed of operation.

Radiation damage produces defects which can result in effects such as [5]:

- Increased dark currents as defects act as centres to increase the generation bulk current;

- Degraded responsivity as defects act as electron or hole trapping centres for the photo-generated pairs, resulting in an increased probability of recombination;

- Degraded rise and fall-times due to de-trapping or a reduction in the carrier mobility.

The increase in the device dark current is expected to be the major change in thin junction devices such as photodiodes. The change in the device response and rise and fall-times of the devices are expected to be small, but still require measuring.

The increase in dark current can be related to the minority carrier lifetime of the semiconductor if the generation-recombination is dominated by mid-band levels caused by defects. Another source affecting the dark current could be ionising damage to the surface of the device, which would generally lead to a catastrophic rise in the current. The dark current, $I_{d c}$, for a device having depletion depth $w$ and active area $A$ is given by,

$$
I_{d c}=\frac{q A w n_{i}}{2 \tau}
$$

where, $q$ is the electron charge,

$n_{i}$ is the intrinsic carrier concentration,

and $\tau$ is the minority carrier lifetime.

The damage process begins with the collision of a high energy incident particle with the semiconductor crystal lattice. The low displacement cross-section of the semiconductor atoms and small volume of semiconductor devices results in a uniform distribution of primary collision sites throughout the material. Furthermore, the recoil neutron will generally exit the device before it undergoes a second interaction. 
The struck atom (known as the primary knock-on atom, PKA) will be knocked from the lattice. It then loses rapidly energy due to both ionisation and the displacement of additional atoms. The division between the ionising and non-ionising damage is well known $[6,7]$. The cascade results in a tree-like damage structure of crystal defects.

The first stage of the damage process is concluded when the PKA comes to rest. The vacancies and interstitials (known together as a Frenkel pair) produced will then diffuse through the semiconductor crystal until they form stable complexes. Many will recombine $(\sim 95 \%)$ leading to an immediate repair of the lattice. However, some will combine to form stable defects such as di-vacancies, vacancy-impurity complexes, vacancy-dopant complexes, and larger clusters. These defects form effective recombination and trapping centres resulting in a decrease in the minority carrier lifetime, carrier density and carrier mobility.

The initial minority carrier lifetime, $\tau_{o}$, can be related to the carrier capture cross-section, $\sigma$, and initial density, $\rho_{o}$, of the centres by the equation,

$$
\frac{1}{\tau_{o}}=\sigma \nu_{t h} \rho_{o}
$$

where $\nu_{t h}$ is the thermal velocity of the minority carriers.

Following irradiation the total minority carrier lifetime, $\tau$, is given by,

$$
\frac{1}{\tau}=\frac{1}{\tau_{0}}+\sigma_{r i} \nu_{t h} \rho_{r i},
$$

where the subscript $r i$ refers to radiation induced centres $(\mathrm{d})$.

If the total number of radiation induced defects is small compared to the size of the crystal, so that damage caused by individual incident particles will not overlap ${ }^{(e)}$, the density of radiation induced non-radiative centres is expected to be proportional to the radiation fluence, $\phi$. It is given by,

$$
\rho_{r i} \equiv C \phi,
$$

where $C$ is constant giving the density of defects produced per unit radiation fluence.

A damage constant $\kappa$ can then be defined,

$$
\kappa \equiv \sigma_{r i} \nu_{t h} C
$$

which, when substituted into equation 3 , yields the relationship between the minority carrier lifetime and the radiation fluence,

$$
\frac{\tau_{0}}{\tau}=1+\tau_{o} \kappa \phi
$$

\footnotetext{
(d) In practice more than one type of defect will be produced and a sum over the capture cross section and density of all the types must be made

(e) For the neutron irradiation of silicon it has been estimated that non-linear effects should not occur before fluence values exceed $\sim 2.5 \times 10^{15} \mathrm{ncm}^{-2}[8]$.
} 
The damage constant parameterises the detailed phenomenological information relating to the physical interactions between the incident particles and the semiconductor material. Rearranging this equation and using equation 1 we can obtain the relationship,

$$
\Delta I_{d c}=q A w n_{i} \kappa \phi
$$

for the radiation induced change in the dark current. The device parameters can be absorbed into the constant by defining a new damage constant,

$$
K=q A w n_{i} \kappa .
$$

The change in the diode dark current is then given by,

$$
\Delta I_{d c}=K \phi
$$

\section{Facilities}

The neutron irradiation of the diodes has been carried out in the Birmingham Dynamitron and Rutherford Appleton Laboratories ISIS facility. The proton irradiation was performed at the CERN Proton Synchrotron.

\section{University of Birmingham Dynamitron}

Neutrons are extracted via the reaction ${ }^{9} \operatorname{Be}(d, n){ }^{10} \mathrm{~B}$, that is, the stripping of deuterons on a thick Beryllium target. The Dynamitron is a variable energy potential drop machine capable of terminal potentials up to $3 M V$, and is powered by a radio-frequency oscillator operating at about $130 \mathrm{kHz}$. For the irradiation tests, a deuteron beam energy of $2.6 \mathrm{MeV}$ and a current of $200 \mu \mathrm{A}$ was used. This gives a neutron energy centred at around $2 \mathrm{MeV}[9]$ with a spread of approximately $2 \mathrm{MeV}$, and a yield of $\sim 10^{11} s r^{-1} s^{-1}$.

Neutron fluences up to $\sim 10^{14} \mathrm{~cm}^{-2} d a y^{-1}$ can be achieved for samples located at small distances from the target, with the Dynamitron operating $\sim 8$ hrs per day.

Activation foils were used to measure the total neutron fluences. Measuring the gamma activity of the foils after irradiation allows the flux to be determined. For the neutron tests, indium foils were cut to sizes corresponding to the components to be irradiated and positioned nearby. The reaction ${ }^{115} \operatorname{In}\left(n, n^{\prime}\right)^{115 m} \operatorname{In}$, with its low threshold of $0.339 \mathrm{MeV}$ and its short lived final state (with a 4.486 hour half-life and $0.459 \mathrm{MeV}$ gamma ray per meta-stable decay) enabled a convenient calibration for these tests. After irradiation, the gamma activity of the foils were measured using a Germanium detector (cooled to $77 K$ ). Typically errors of $\sim 10 \%$ were obtained in the measurement of activity; these were due mainly to the limited time of the measurement. This is the dominant uncertainty in the fluence measurement. 


\section{ISIS Radiation Hardness Test Facility}

The spallation neutron source ISIS [10] at the Rutherford Appleton Laboratory (RAL), UK has an energy spectrum centred around $1 \mathrm{MeV}$. Neutron fluxes between $1.7 \times 10^{7}$ and $5 \times 10^{8} \mathrm{~cm}^{-2} \mathrm{~s}^{-1}$ are possible. The received exposure of the irradiated devices is monitored using cobalt foils.

\section{CERN Proton Synchrotron}

The proton irradiation studies used the CERN Proton Synchrotron (PS), Geneva. The proton energy is $24 \mathrm{GeV}$ and fluences of $10^{14} \mathrm{~cm}^{-2} \mathrm{day} y^{-1}$ are easily obtainable. (Typically there are bunches of $10^{11}$ protons every 14 seconds; the spread is dependent on the beam optics but an area $1 \mathrm{~cm} \times 1 \mathrm{~cm}$ is achievable). In these irradiation tests the devices under test were mounted on an $\mathrm{x}-\mathrm{y}$ stage which scans an area of $6 \mathrm{~cm} \times 6 \mathrm{~cm}$ transverse to the beam direction (designed for the irradiation of silicon detectors). The PS is operational 24 hours a day. Consequently, expected fluences are of the order of $10^{13} \mathrm{~cm}^{-2} \mathrm{day} \mathrm{y}^{-1}$.

For the proton tests, aluminium foils were used for an initial calibration run. The fluences obtained, via the reaction ${ }^{27} \mathrm{Al}(\mathrm{p}, 3 \mathrm{pn}){ }^{24} \mathrm{Na}$ (producing a $1.368 \mathrm{MeV}$ gamma with a $\sim 15$ hour half-life), were used to calibrate a Secondary Emission Counter. The activity of the aluminium foils was measured in the same way as for the indium foils. The errors on the activity measurements were of comparable size.

\section{Scaling of Damage}

The damage in Si devices results predominantly from the non-ionising inelastic energy lost (NIEL) by the incident particles to the semiconductor material. This has been verified experimentally for different incident particles, over a wide range of incident energies, for $\mathrm{Si}$ [11-14]. Therefore, the expected particle fluences can be expressed in terms of effective fluences of particular particles with fixed energy. Two standard particle types are chosen: $1 \mathrm{MeV}$ equivalent neutrons and $24 \mathrm{GeV}$ equivalent protons. The NIEL stopping powers for the standard particle types are given in Table 1 together with those for the radiation sources used in testing the components. The latter were calculated by integrating data from references $[11,13]$ over the measured energy spectra. The uncertainty on the values given for neutron data is $\sim 10 \%$, while for proton data an uncertainty $\sim 20 \%$ is not unlikely.

Table 1: NIEL stopping powers for various particle types in $\mathrm{Si}\left(k e V\left(\mathrm{~g} \mathrm{~cm}^{-2}\right)^{-1}\right)$.

\begin{tabular}{l|c} 
Irradiation & NIEL \\
\hline $1 \mathrm{MeV}$ neutrons & 1.80 \\
$24 \mathrm{GeV}$ protons & 1.0 \\
ISIS neutrons & 2.17 \\
Dynamitron neutrons & 1.97
\end{tabular}


The integrated fluences (for the 10-year lifetime of the ATLAS experiment, assuming the usual ATLAS luminosity scenario $\int L d t=7.3 \times 10^{5} \mathrm{pb}^{-1}$ ) at the radius of the smallest SCT barrel are given in table 2. The fluences are expressed in terms of $1 \mathrm{MeV}$ equivalent neutrons and $24 \mathrm{GeV}$ equivalent protons in Silicon. Irradiation of components by both protons and neutrons is required for confidence. Irradiation by charged particles carries an increased likelihood of ionization damage resulting in, for instance, surface charge effects.

Table 2: Equivalent fluences at the $1^{\text {st }} \mathrm{SCT}$ barrel $\left(\times 10^{13} \mathrm{~cm}^{-2}\right)$.

\begin{tabular}{l|c|c} 
Particle Type & $F_{n(1 \mathrm{MeV})}$ & $F_{p(24 \mathrm{GeV})}$ \\
\hline Charged particles & 6.3 & 11.3 \\
Neutrons & 6.7 & 12.1 \\
Total & 13.0 & 23.4
\end{tabular}

\section{$5 \quad$ Results}

\subsection{Neutron Irradiation}

The basic scheme of the experimental setup is shown in Figure 3. The photodiodes were biased at $-10 \mathrm{~V}$ during the tests. This was sufficient to fully deplete the devices and is the expected reverse bias required for fast operation in the readout. A $100 \mathrm{k} \Omega$ series resistor allowed the current through the diode to be monitored. The dark current, when no light was incident on the photodiode, and the photocurrent for incident light powers from $3-20 \mu W$ in five equal steps was measured. From these measurements the response of the photodiode was determined. The first step is to make a $3^{\text {rd }}$ order polynomial fit to the incident light power versus LED drive current. This is used to calculate the incident light on the photodiode for a given LED drive current $^{(f)}$. A similar fit is then made to the photocurrent vs incident light power points. The response is calculated from the slope of the fitted function for a launched optical power of $10 \mu W$ into the photodiode fibre.

\section{Preliminary Bulk Silicon Photodiode Irradiation}

The first tests were performed with three bulk Si PIN-diodes mounted in the LED/PIN packages shown in 2 . An overview of the results from these tests is given before more detailed discussion of the tests on a larger sample of photodiodes.

During the proton irradiation the photodiode response was measured, but not the dark current. The photodiodes were exposed to a total proton fluence of $0.4 \times$

(f) The incident light power is measured at the launch point into the fibre patchcord running between the barracks and photodiode. The incident light on the photodiode can be expected to be systematically lower than the measured value (by $\sim 5 \%$ ) due to loss in the fibre and at connectors. However, we are more interested in changes in the response. 


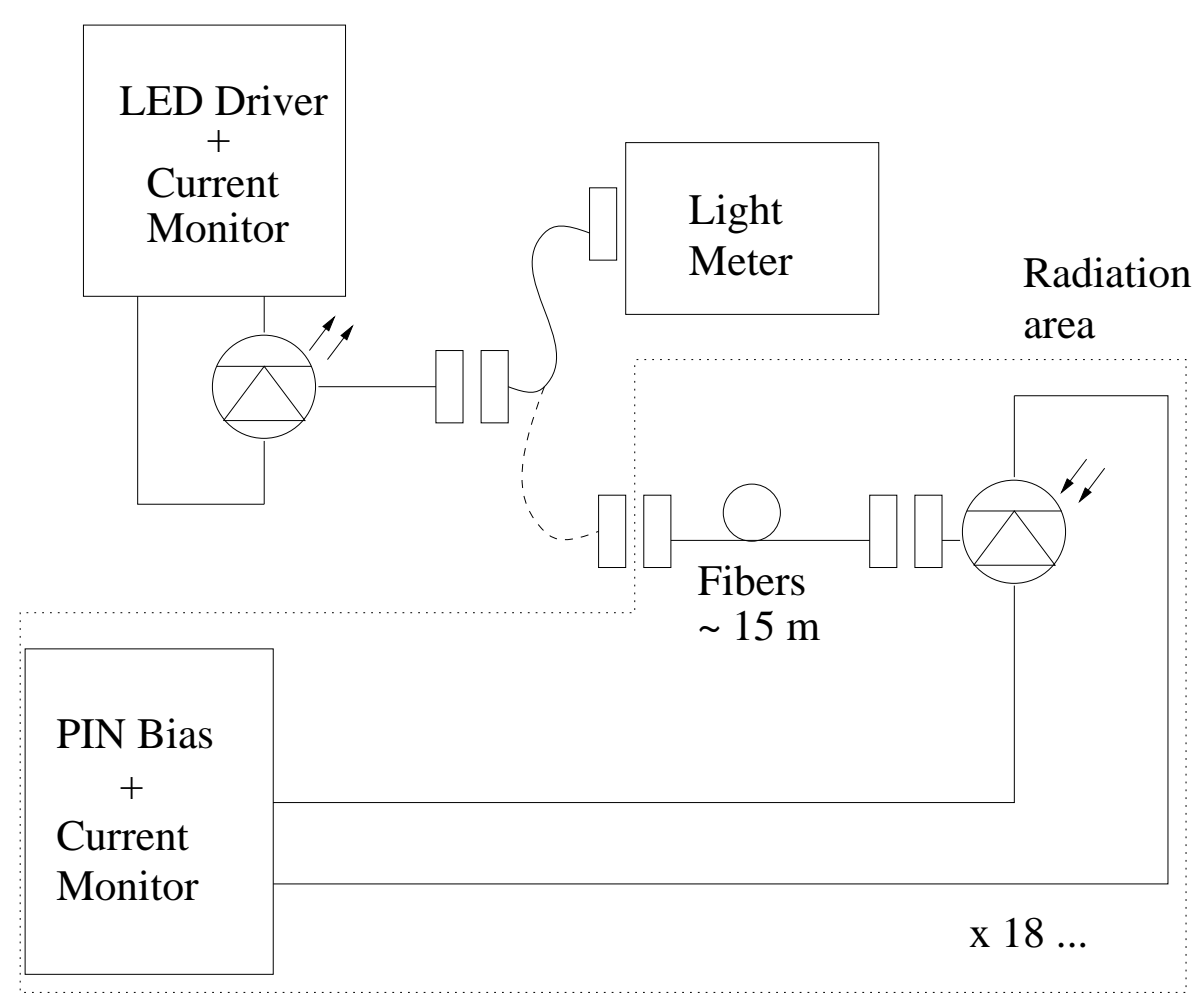

Figure 3: Diagram of the experimental setup.

$10^{14} \mathrm{~cm}^{-2}$. The response of the photodiodes was measured before the exposure began when the diodes were at both the beam area temperature, $30^{\circ} \mathrm{C}$, and the nominal coolbox temperature, $-7^{\circ} \mathrm{C}$. In both cases the response of all three photodiodes was measured to be $\sim 0.5 \mathrm{~A} / \mathrm{W}$. The three photodiodes responded in different ways during the exposure period. The response of one photodiode dropped by only $\sim 10 \%$ while another fell to $0.1 \mathrm{~A} / \mathrm{W}$, only $20 \%$ of the starting value, and the third did something in between. However, during a short stop in the irradiation, while the coolbox dewar was refilled, and following the termination of the irradiation all three photodiodes recovered to close to their pre-irradiation values over a period of a day. The annealing is correlated with a rise in temperature to that of the surroundings in each case. It is hard to draw quantitative conclusions on the performance of the diodes from these three different cases, however the large drop seen by one photodiode is by itself worrying.

Following the return of the devices to Birmingham the photodiodes were exposed to neutron fluences in excess of $2 \times 10^{14} \mathrm{~cm}^{-2}$. During these tests the monitoring was changed to allow the dark current to be measured in addition to the response of the diodes. The photodiodes behaved in a consistent, but disappointing, manner during these tests. All three devices showed a rapid fall in response to $<0.1 \mathrm{~A} / \mathrm{W}$ after only a short period of irradiation. No subsequent recovery was measured. The dark currents of the diodes was measured at $\sim 15 n \mathrm{~A}$ before the neutron irradiation. This shows a deterioration from the expected pre-irradiation value of $<1 n A$ due to the proton exposure. The dark current rose steadily throughout the neutron exposure 
to a final value of $\sim 0.8 \mu \mathrm{A}$ after the full neutron fluence. The dark current dropped and leveled out at $\sim 0.5 \mu \mathrm{A}$ over the two day period following the irradiation.

The increase in the dark current although large is well behaved and does not indicate a catastrophic failure in the device. However, the large decrease in the response of the photodiodes due to the neutron irradiation is not expected. Further tests on photodiodes have been performed and the results are discussed in more detail below.

\section{Bulk Silicon Photodiode Irradiation}

In order to perform further tests of bulk Si PINs and to investigate epitaxial PINs which were expected to be more radiation tolerant, packages similar to those shown in Figure 2 were made containing three PIN diodes instead of two LEDs and one PIN. The experimental set-up consisted of a PCB board on which were glued six packages: three packages contained bulk silicon diodes and other three epitaxial diodes (see further). The connection between the PIN-diode signal and the PCB track was made by a wire bond. A yield of eight working bulk silicon photodiodes from the possible nine was achieved. The one diode not working was due to a bad wire bond giving no electrical connection rather than a problem with the device itself.

Neutron irradiation of these devices has been carried out using the Birmingham Dynamitron. The running schedule consisted of three $\sim 8$-hour long daytime irradiations, yielding $1 \mathrm{MeV}$ equivalent neutron fluences of around $1.7 \times 10^{14} \mathrm{~cm}^{-2}$, followed by twelve days monitoring with the devices still in the test area.

The results of the irradiation on the photodiodes is shown in figure 4. The response of all the diodes drops rapidly during the first irradiation period. The plot of relative response versus the fluence highlights this rapid fall, induced by a neutron fluence of only $\sim 0.25 \times 10^{14} \mathrm{~cm}^{-2}$, to a relative response of only $10 \%$. The degradation saturates at this level for the remainder of the irradiation and no post-irradiation recovery is observed. The dark current of the diodes shows a large increase over the irradiation period. The initial dark current for all the diodes was too small to measure and consistent with the $<1 n A$ value quoted by the supplier. The final value was $\sim 500 \mathrm{nA}$ immediately after the irradiation dropping to $\sim 300 \mathrm{nA}$ after several days. Equation 9 predicts a linear increase in the dark current with fluence which can be characterised by the damage constant $K_{\text {bulk }}$. The annealing between the three irradiation periods, and presumably during the irradiation, probably invalidates any fit to the data. However, if only the final value just after the finish of the irradiation is considered a damage constant $K_{\text {bulk }} \approx 3.3 \times 10^{-12} \mathrm{nA} \mathrm{cm}^{2}$.

An additional neutron irradiation has been carried out in the ISIS facility [10]. $1 \mathrm{MeV}$ equivalent neutron fluences of $7.9 \times 10^{13} \mathrm{~cm}^{-2}$ were achieved over a 38 day irradiation period. The response of the diodes was unchanged. The dark currents increased linearly as expected. 


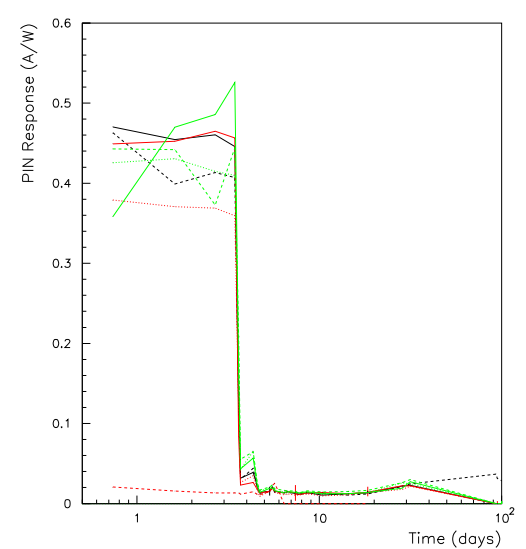

(a) Response vs Time.

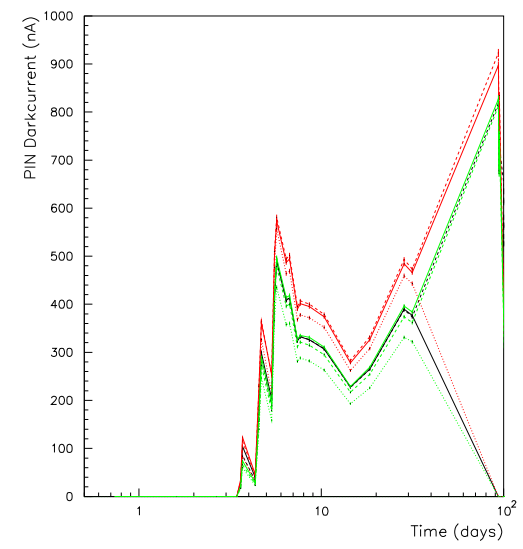

(c) Dark current vs Time. The points at zero after the ISIS irradiation correspond to broken wire bonds.

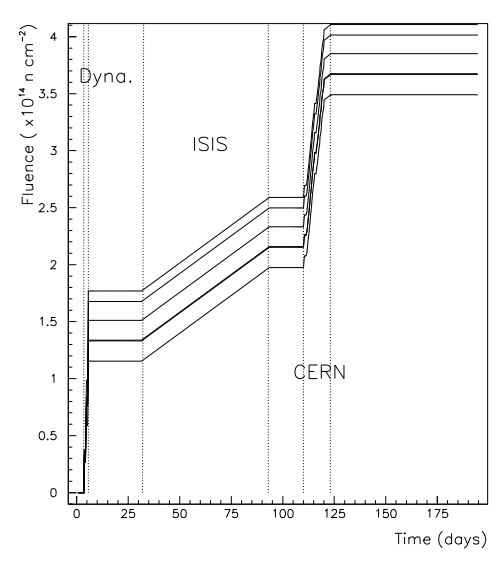

(e) Fluence vs Time.

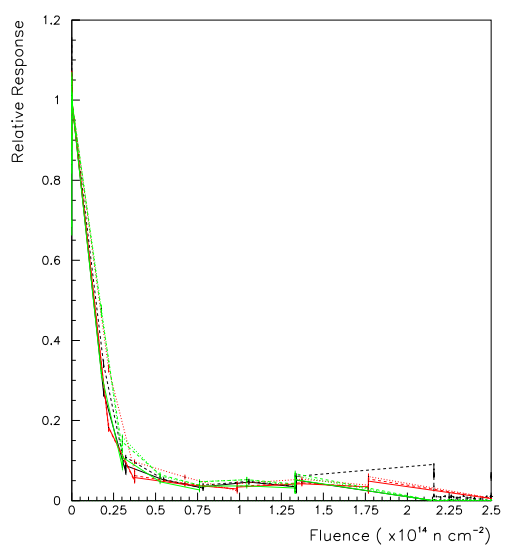

(b) Relative response vs Fluence.

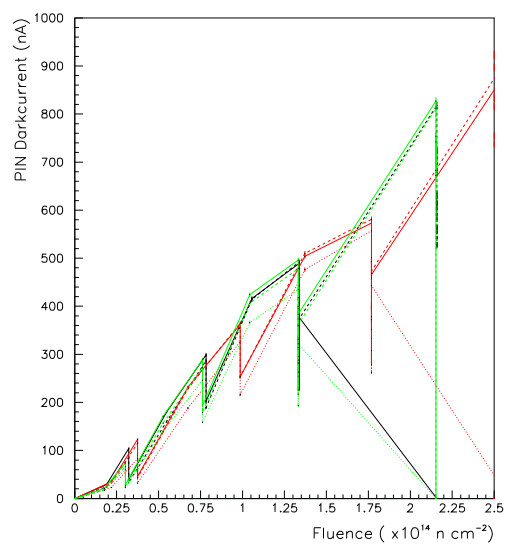

(d) Dark current vs Fluence.The vertical lines correspond to periods of time over which the devices were not being irradiated.

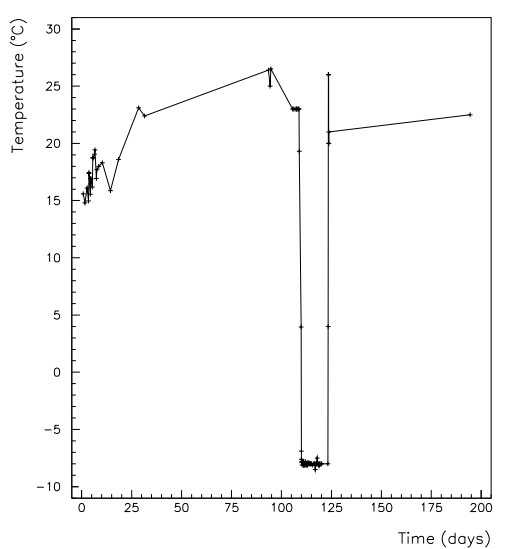

(f) Temperature vs Time.

Figure 4: Results of bulk silicon photodiode neutron irradiation. 


\section{Epitaxial Silicon Photodiode Irradiation}

The epitaxial Silicon photodiodes were irradiated at the same time as the bulk Silicon diodes. The measurements and analysis of the data were performed in exactly the same way as previously described. Only seven were tested due to broken wire bonds, one of them during the experiment.

The results of the irradiation tests are shown in figure 5. The response of the diodes exhibits the same immediate drop followed by stability as the bulk photodiodes, but with the important distinction that stable behaviour occurs after a fall of only $\lesssim 30 \%$. The dark current rises throughout the irradiation as expected with a damage constant $K_{\text {epitaxial }} \approx 0.13 \times 10^{-12} \mathrm{nA} \mathrm{cm}^{2}$, ignoring the annealing.

The additional neutron irradiation in the ISIS facility resulted in no change in the response of the diodes. The increase in dark currents followed the same slope as previously observed.

\subsection{Proton Irradiation}

The epitaxial diodes have been subsequently irradiated with $24 G e V$ protons in the CERN PS to fluences of $2.5 \times 10^{14} \mathrm{p} \mathrm{cm}^{-2}$. The results are shown on figure 6 and the corresponding $1 \mathrm{MeV}$ equivalent neutron fluence period is from $2.5 \times 10^{14} \mathrm{n} \mathrm{cm}^{-2}$ to $4 \times 10^{14} \mathrm{~cm}^{-2}$ (fig. 5e). The PIN diodes were cooled to $-8^{\circ} \mathrm{C}$, decreasing the darkcurrent to less than $5 \mathrm{nA}$. The damage constant at this temperature becomes $K_{\text {epitaxial }}^{-8^{\circ}} \approx 0.017 \times 10^{-12} \mathrm{nA} \mathrm{cm}^{2}$. At ambient temperature, the dark current resumes its initial rise. The responsivity of the epitaxial PIN diodes remains stable at about $0.25 \mathrm{~A} / \mathrm{W}$ with no further sign of degradation.

\subsection{Possible Interpretation}

One explanation for this initial response degradation could be type inversion of the device substrate $[15,16]$. The incident radiation causes the formation of acceptor levels which result in the low-doped n-type region changing to a low-doped p-type region. The result is that the depletion region now grows from the p-type side of the diodes rather than the n-type side. The distance for absorption of $99 \%$ of $820 \mathrm{~nm}$ light in Silicon is only $\sim 45 \mu \mathrm{m}$ and so it is possible that the light will not reach the depletion region in the bulk Si devices if the devices are not fully depleted.

Figure 7 shows the response and dark current for typical bulk and epitaxial diodes after neutron irradiation as a function of the applied bias voltage. The response of a bulk diode before irradiation is included for comparison. The response has been calculated using the method described above. It can be seen that the response of the epitaxial diode and the bulk diode before irradiation quickly reach a plateau at a low reverse bias. This indicates that the epitaxial device is fully depleted and the bulk device is depleted at least as deep as $\sim 45 \mu \mathrm{m}$. It can also be observed that the epitaxial diode dark current approaches a plateau again indicating full depletion. However, the bulk diode after irradiation shows neither of these attributes. The response rises approximately linearly with the applied voltage and the dark current is still increasing at a bias voltage of $-20 \mathrm{~V}$, the maximum specified voltages for the 


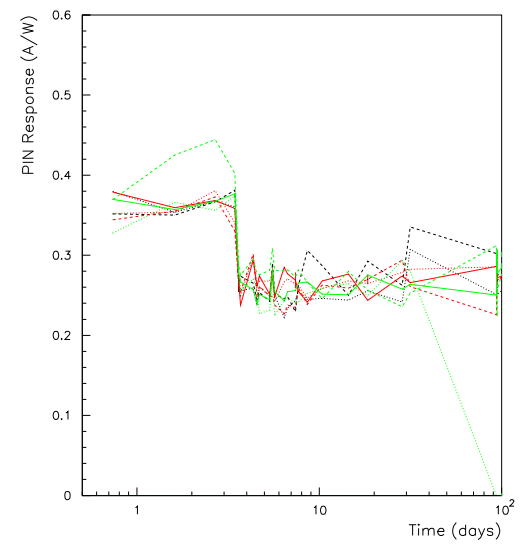

(a) Response vs Time. Note the fall in response after 3 days due to the first exposure to radiation. The point at zero corresponds to a broken wire bond on the device.

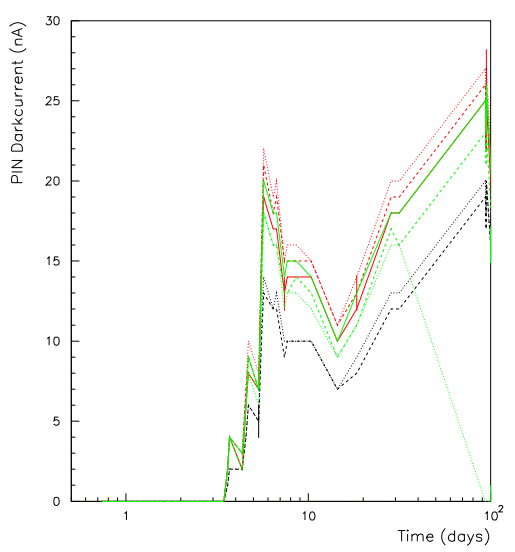

(c) Dark current vs Time.

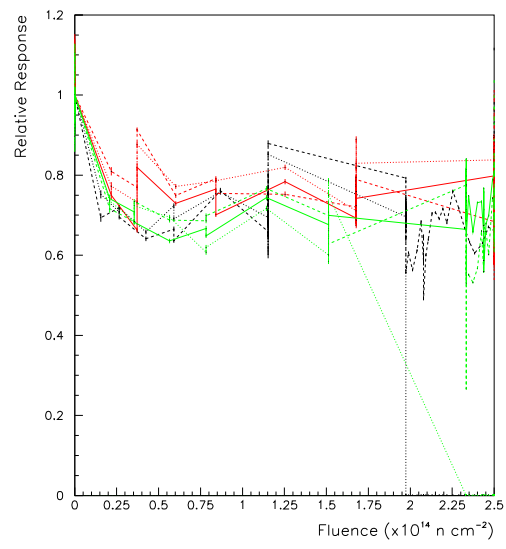

(b) Relative response vs Fluence. The vertical lines correspond to periods over which the devices are not being irradiated.

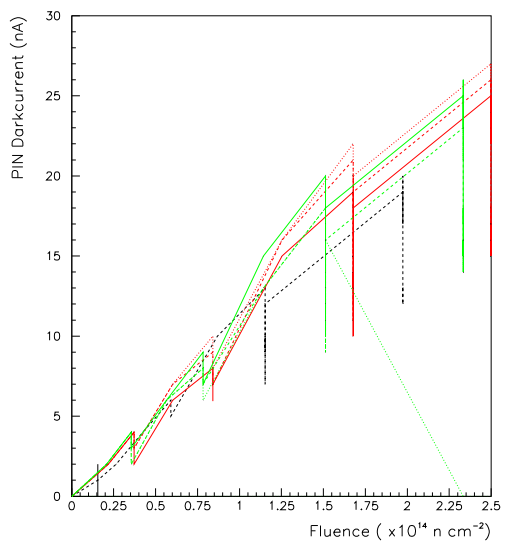

(d) Dark current vs Fluence.

Figure 5: Results of epitaxial silicon photodiode neutron irradiation. 


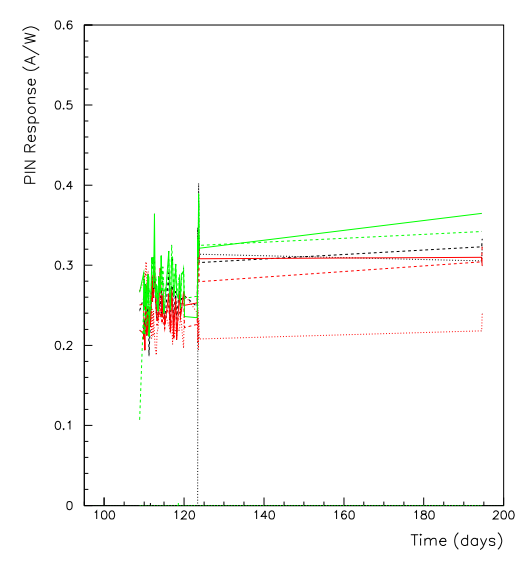

(a) Response vs Time.

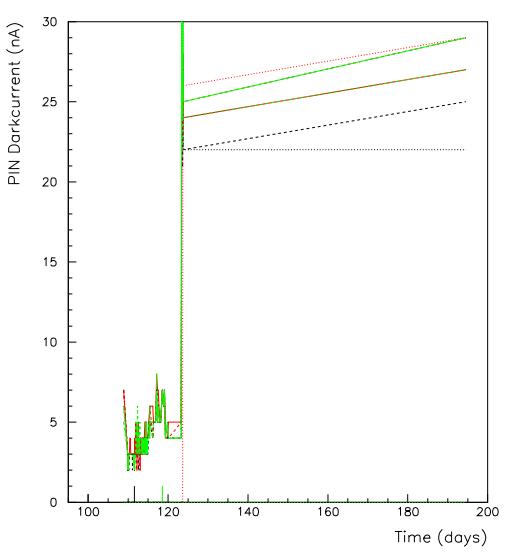

(c) Dark current vs Time. During the $\mathrm{p}$ irradiation (days 107 124) the devices were held at $-8^{\circ} \mathrm{C}$. Afterwards ( $>124$ days), they were at room temp.

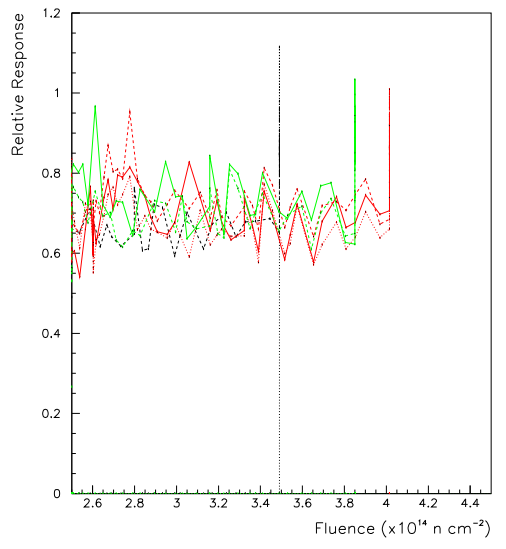

(b) Relative response vs Fluence.

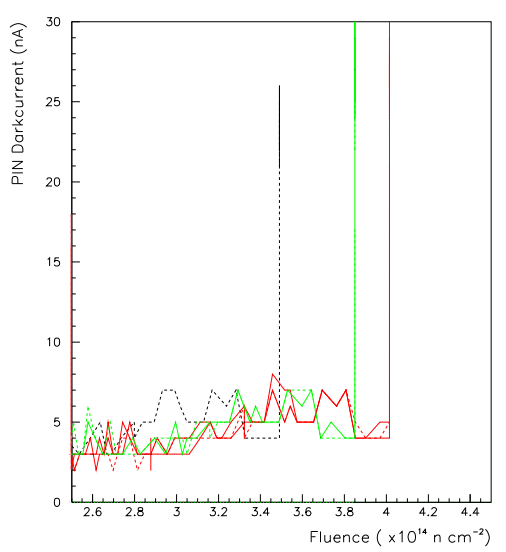

(d) Dark current vs Fluence. The devices were held at $-8^{\circ} \mathrm{C}$ during irradiation; then after irradiation, at room temp.

Figure 6: Results of epitaxial silicon photodiode proton irradiation. 
diodes. These results indicate that the bulk diodes are not being fully depleted after neutron irradiation.

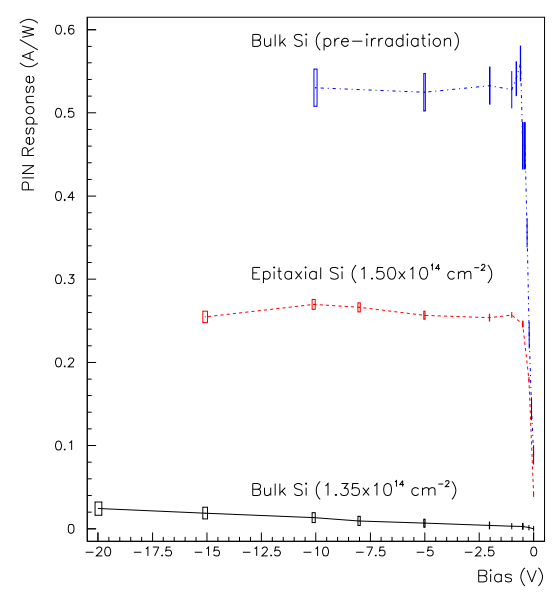

(a) Response.

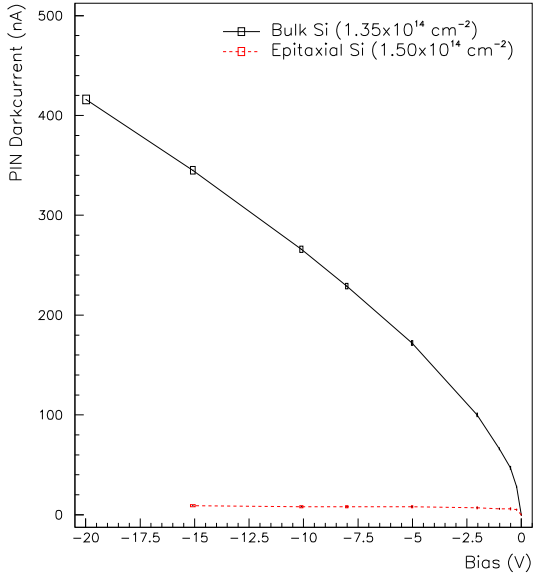

(b) Dark current.

Figure 7: Measurement of the photodiode voltage characteristics after irradiation.

\subsection{Rise and Fall time of Irradiated PIN diodes}

The manufacturer's specifications for the epitaxial Si PIN diodes quote rise and fall times of less than 1 ns. It is important to verify that this is not significantly degraded after irradiation. The rise and fall times for an epitaxial Si PIN diode were measured after it had been irradiated to the total $1 \mathrm{MeV}$ equivalent neutron fluence of $4 \times 10^{14} \mathrm{~cm}^{-2}$. The PIN diode was illuminated with light from a VCSEL (Honeywell HFE 4080) and the signal was measured using a simple circuit with purely passive components. The VCSEL drive current was adjusted to give about $1 \mathrm{~mW}$ of optical power but it was checked that the rise and fall times were insensitive to the magnitude of the optical power. The rise and fall times were measured (using a digital oscillscope) as a function of the PIN bias voltage and the results are shown in Fig.8. The measured rise and fall times should be regarded as upper limits on the rise and fall times of the PIN diode because they include contributions from the VCSEL drive circuitry, the VCSEL itself and the finite speed of the oscilloscope. ¿From Fig. 8 it is clear that excellent rise and fall times of the irradiated PIN diodes can be obtained provided the bias voltage is greater than about $5 \mathrm{~V}$.

\section{Conclusions}

The dark current and response of both bulk silicon and epitaxial silicon photodiodes have been measured throughout irradiation with neutrons and protons. Approximate damage constants have been found for the increase in dark current for the de- 


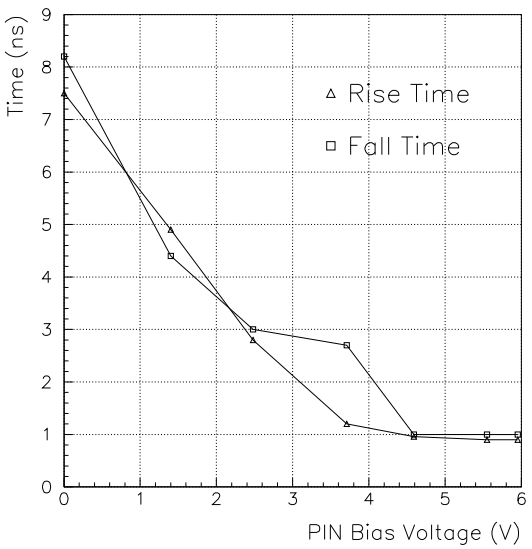

Figure 8: Measured rise and fall times of the received signal from the irradiated epitaxial Si PIN diode versus bias voltage. The lines drawn are to guide the eye only.

vices; the values are $3.3 \times 10^{-12} \mathrm{nA} \mathrm{cm}^{2}$ for the bulk Si diodes and $0.13 \times 10^{-12} \mathrm{nA} \mathrm{cm}^{2}$ for epitaxial $\mathrm{Si}$ diodes when scaled to $1 \mathrm{MeV}$ neutrons. After the fluence expected for the lowest radius SCT barrel of $1.3 \times 10^{14} \mathrm{~cm}^{-2} 1 \mathrm{MeV}$ equivalent neutrons, the predicted dark currents are $\sim 390 n A$ and $\sim 16 n A$ for the bulk and epitaxial Si devices respectively. The dark current damage in the epitaxial $\mathrm{Si}$ devices is much lower due to the $\sim 10 \times$ smaller volume of the devices ${ }^{(\mathrm{g})}$.

The response of both types of diode decreases after neutron fluences of only $\sim 0.25 \times 10^{14} \mathrm{~cm}^{-2}$. However, after the initial drop the diodes response is then unaffected for $1 \mathrm{MeV}$ equivalent neutron fluences up to $\gtrsim 2 \times 10^{14} \mathrm{~cm}^{-2}$ as expected. The initial fall in the response of the bulk Si diodes is fatal. However, the epitaxial diodes exhibit a fall of $\lesssim 30 \%$ which is acceptable for use in the final package. Moreover, the measurements of the rise and fall times of the diodes after irradiation confirm that the devices are suitable for the SCT readout. They are also good candidates for use with the pixel detetectors.

\section{Acknowledgements}

We thank the Birmingham technicians Roger Harris and Ian McGill. We would like to thank the whole ATLAS SCT irradiation group and in particular Craig Buttar for the success of the PS irradiation. We are grateful to the staff of the University of Birmingham Dynamitron, and to Mike Edwards and Derrick Hill at RAL for ISIS tests. We also thank Dr. Jon Hall (GEC-Marconi) for very useful discussions on PIN diode radiation hardness. The work was supported by the UK Particle Physics and Astronomy Research Council.

(g) The depletion region thicknesses are $\sim 15 \mu \mathrm{m}$ for the epitaxial diodes and $\sim 100-150 \mu \mathrm{m}$ for the bulk devices. The difference in volumes will therefore be of the order a factor 10 . 


\section{References}

[1] ATLAS Inner Detector Community, "ATLAS Inner Detector Technical Design Report”, CERN Report CERN/LHCC/97-16 (1997).

[2] C.B.Brooks et al., "Optical Links for the ATLAS SCT", Proceedings of the First Workshop on Electronics for the LHC Experiments, Lisbon, 1995, CERN Report CERN/LHCC/95-56 (1995).

[3] J.Beringer et al., "Radiation Hardness and Lifetime Studies of LEDs and VCSELs for the Optical Readout of the ATLAS SCT", ATLAS Internal Note, INDET-NO-183, September 1997.

[4] S.Oglesby, "Investigation of Opto-electronic Data Transmission Methods for Experiments at the CERN Large Hadron Collider", PhD Thesis, University of Birmingham 1997.

[5] H.W.Kraner, Nucl. Inst. and Meth. 225, 615 (1984).

[6] J.Lindhard et al., "Range concepts and heavy ion ranges", Kgl. Dan. Vidensk. Selsk. Mat.-Fys. Medd. 33, 2 (1963).

[7] J.Lindhard et al., "Integral Equations Governing Radiation Effects", Kgl. Dan. Vidensk. Selsk. Mat.-Fys. Medd. 33, 3 (1963).

[8] G.C.Messenger, IEEE Trans. Nucl. Sci. 39, 468 (1992).

[9] J.W.Meadows, Nucl. Inst. and Meth. in Phys. Res. A324, 239 (1993).

[10] M.Edwards and D.R.Perry, "The Radiation Hardness Test Facility", Rutherford Appleton Laboratory Report RAL-90-065.

[11] T.F.Luera et al., IEEE Trans. Nucl. Sci. NS-34, 1557 (1987).

[12] A. van Ginneken, "Non Ionizing Energy Deposition in Silicon for Radiation Damage Studies", FERMILAB report FN-522 (1989).

[13] A.M.Ougouag IEEE Trans. Nucl. Sci. NS-37, 2219 (1990).

[14] M.Huhtinen and P.A.Aarnio, Nucl. Instr. and Meth. A335, 580 (1993).

[15] N.Tamura et al., Nucl. Inst. and Meth. in Phys. Res. A342, 131 (1994).

[16] D.Pitzl et al., Nucl. Inst. and Meth. in Phys. Res. A311, 98 (1992). 